EDITORIAL

\title{
Can we ablate permanent atrial fibrillation?
}

G Haywood

Heart 2006;92:152-154. doi: 10.1136/hrt.2005.075945

There is growing evidence of the benefit of ablation in the treatment of drug refractory atrial fibrillation

A trial fibrillation (AF) used to be considered a rather uninteresting arrhythmia; now it is firmly centre stage. In the article by Earley and co-workers ${ }^{1}$ in this issue of Heart, it seems that the ablation of even permanent AF has become a legitimate goal for cardiac electrophysiologists. Should we be gearing up to ablate all drug refractory symptomatic AF?

Permanent AF is defined as either the inability to achieve cardioversion to sinus rhythm, or the recognition that attempts to do so would be futile. Until recently it has represented the end point in the natural history of the progression of AF as a rhythm disorder, from either newly diagnosed paroxysmal AF (with spontaneous reversion to sinus rhythm within seven days) or persistent AF (where electrical or pharmacological cardioversion is required). ${ }^{2}{ }^{3}$ Increasingly it is apparent that acceptance of this end point need not be inevitable.

\section{RESTORING SINUS RHYTHM}

The proof that medical intervention can restore sinus rhythm to patients with permanent AF on a long term ( 15 year $^{4}$ ) basis originally came from the cardiac surgical literature. The Cox Maze procedure, with a cure rate $>95 \%$, ${ }^{5}$ has gone through a series of modifications and has been reduced to a minimal lesion set that seems to preserve the very high level of efficacy of the earlier more complex versions. The key elements appear to be ${ }^{6}$ :

- encircling of the pulmonary veins, either (1) as a single circle around all four veins or (2) as a pair of encircling ellipses, one around the right pulmonary veins and the other around the left, joined by a line of block linking their inferior margins

- a line linking the inferior margin of the above lesions to the mitral valve annulus

- a circumferential lesion in the coronary sinus musculature at the point that the line links to the mitral annulus

- a "right atrial isthmus" line from the tricuspid annulus to the inferior vena cava (IVC) (fig 1).

Correspondence to:

Southwest Cardiothoracic Centre, Derriford Hospital Plymouth PL6 8DH, UK; guy.haywood@phnt.swest. nhs.uk

Published Online First 24 November 2005 .................... minimal lesion set above and electroanatomic mapping to validate the presence of complete block across the lesions ( $86 \%$ in sinus rhythm off drugs at two years in patients with permanent AF undergoing valvar surgery ${ }^{9}$ ).

This "minimal" lesion set closely resembles the approach used by Earley et al $^{1}$ and others who have reported encouraging levels of curative results using the percutaneous approach in subsets of patients with permanent AF $(76 \%$ at 8.4 months, ${ }^{1} 72 \%$ at 15 months $^{10}$ ). Some of the groups with the largest and most influential series have reported $80 \%$ success rates ${ }^{11}$ with this type of percutaneous approach for "chronic AF", but have not published detailed reports that separate out patients with permanent AF from those with persistent AF. Others have reported similar, or even better, percutaneous success rates in patients with permanent $\mathrm{AF}$ with still simpler lesion sets. ${ }^{12} 13$

The main difference between the surgical cutand-sew technique and the percutaneous approaches seems to be the reliability of the permanence of the line of electrical block and also the reduction in size of the remaining sectors of electrically intact atrial myocardium. Using the cut-and-sew approach the surgeon can reduce the surface area of the left atrium leaving smaller sectors in which a driving rotor ${ }^{14}$ might re-establish itself in the remodelled atrial myocardium. ${ }^{15}$ There is also no significant chance of cells recovering within the line of block, whereas with radiofrequency and microwave ablation there is the potential for myocytes to survive apparently transmural lesions and re-establish conduction. This may in part relate to the protective effect of cooling by blood flow in small intramyocardial vessels. ${ }^{16}$ This may explain why even when performed intraoperatively, cure rates are lower using these modalities $\left(62 \%{ }^{17}\right.$ to $81 \%^{18}$ of permanent AF patients in sinus rhythm at one year with microwave, and $44.4 \%{ }^{19}$ to $80 \%{ }^{18}$ with radiofrequency energy).

\section{PREVENTING INDUCTION OF AF}

Non-remodelled myocytes, such as are found in paroxysmal and short term persistent $\mathrm{AF}$, have a much lower propensity to sustain AF than those found in permanent AF. ${ }^{15}$ In patients with paroxysmal or persistent $\mathrm{AF}$, procedures that result in conduction delay and functional block when electrical triggers in the pulmonary veins fire at high rates may be sufficient to prevent induction of AF. Curative outcomes, despite incomplete isolation of the initiating triggers, are facilitated by the adjunctive use of antiarrhythmic drugs in such patients..$^{20}$ In permanent $\mathrm{AF}$, however, recurrence seems almost guaranteed in the absence of complete electrical 


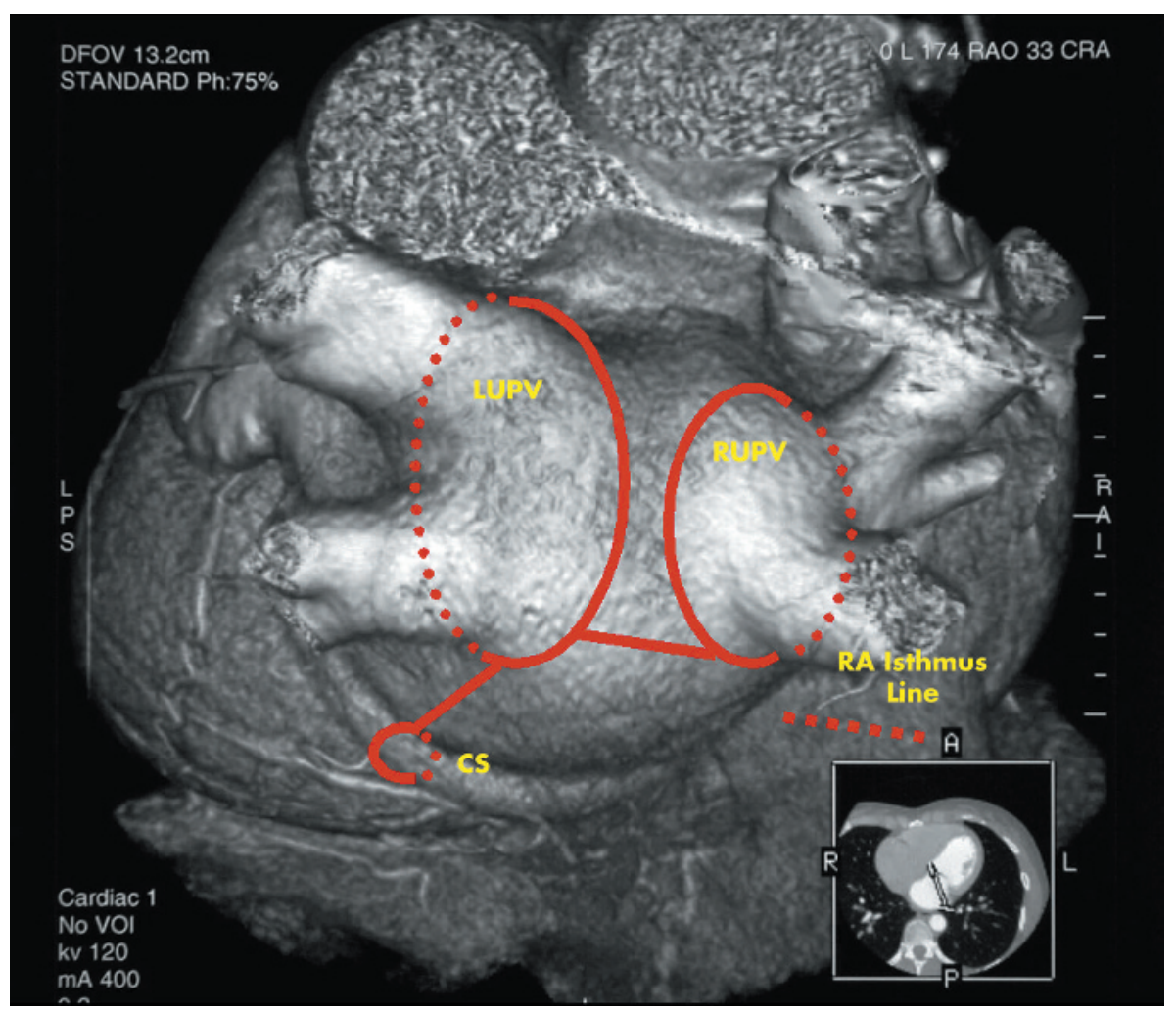

Figure 1 Computerised tomographic angiogram of the posterior aspect of the left atrium. Red lines indicate lines of electrical conduction block in a modified version of the Cox procedure. CS, coronary sinus; LUPV, left upper pulmonary vein; RUPV, right upper pulmonary vein; $R A$ isthmus line, right atrial isthmus line.

block. As a result the experience reported here by Earley et al ${ }^{1}$ and by others, including Haissaguerre and co-workers, ${ }^{21}$ is that up to three or even more procedures are needed to finally achieve a cure. Almost universally the reported finding at repeat procedures is of reconnection across a previously documented line of electrical block between the triggering source and the atrial myocardial substrate. ${ }^{22}$

The main challenge seems to be to deliver enough energy to achieve permanent transmural ablation without incurring serious complications. Intraoperative cryoablation has a good track record of safety and efficacy, but when used percutaneously requires a relatively large calibre catheter for effective lesion creation and procedure times are prohibitively long. ${ }^{23} 24$ With radiofrequency energy there is little to indicate when sufficient power has been delivered to achieve permanent transmural ablation. The therapeutic range separating efficacy from "toxicity" is narrow. Haissaguerre and colleagues limit power to $35 \mathrm{~W}$ to avoid cardiac perforation, ${ }^{25}$ but this is probably at the lower limit of what is necessary to achieve transmural lesions in thick myocardium. The problem is compounded by the extreme variability of atrial myocardial wall thickness at key areas such as the "mitral isthmus" where myocardial thickness in cadaveric human hearts is seen to vary from $0-7.7 \mathrm{~mm}^{26}$ Endocardial electrogram amplitude, the formation of double potentials, and a fall in impedance are of some value, but imprecise.

\section{MULTIPLE PROCEDURES}

The present situation is that for patients in permanent AF a cure rate of $>70 \%$ with serious complications occurring in less than 3\% can be achieved by the most skilled and dedicated groups. Curative treatment will, however, require multiple procedures in around half the patients. The big question is to what extent this technical ability, demonstrated in a few centres around the world, can and should be extended to a great many more centres to meet the potential population demand.
Given the 314 minute mean procedure time in the series reported here ${ }^{1}$ and the rates given for second and third procedures, the mean total catheter laboratory time to achieve a cure for a patient with permanent AF works out at around eight hours. There are very little data giving population estimates of the demand for AF ablation. In the 1998 North American Society for Pacing and Electrophysiology (NASPE) expert consensus report ${ }^{27}$ an attempt was made to project the workforce requirement for ablation in the USA. This document estimated that there are $>1000 /$ million population currently requiring AF ablation, with 180/million population new cases arising and requiring AF ablation each year. This was clearly long before the prospect of ablating permanent AF using a percutaneous approach became realistic and probably represents an underestimate given the results reviewed above. Using these estimates, how much catheter laboratory time are we likely to need to meet the demand for AF present in the population? If we assume that an AF ablation for paroxysmal or persistent AF takes a mean of 3.5 hours (including an allowance for redo cases), and that permanent AF cases will make up $35 \%{ }^{11}$ of AF ablation cases, the time spent ablating new incident cases per year would be 19 hours per week. Additionally, if the backlog of AF cases already present were to be cleared in five years, an extra 21 hours per week of activity over the first five years would be required (calculations based on a 48 week working year). A total of 40 hours of continuous catheter laboratory time per million population would therefore need to be dedicated to AF ablation. This is probably a conservative estimate.

What seems certain is that, as patients and their physicians read that curative results can be obtained for drug refractory, symptomatic $\mathrm{AF}$, including permanent $\mathrm{AF}$, a high percentage of them will wish to undergo these treatments. Although prognostic and quality of life data from long term randomised trials of catheter ablation for AF are still in preparation, the non-randomised data comparing ablation to 
continued medical treatment suggests a strong benefit from ablation..$^{28}$ Unless we find a much quicker way of achieving a cure, we are going to need a lot more catheter laboratories and highly trained staff to deliver the benefits demonstrated by Earley et al ${ }^{1}$ to a wider population.

Conflicts of interest: The author holds a patent related to atrial fibrillation ablation. No other conflicts of interest.

\section{REFERENCES}

Earley MJ, Abrams DJR, Staniforth AD, et al. Catheter ablation of permanent atrial fibrillation: medium term results. Heart 2006;92:233-8.

2 McNamara RL, Brass LM, Droxda JP et al for the ACC/AHA Data Standards on Atrial Fibrillation Task Force. ACC/AHA key data elements and definitions for measuring the clinical management and outcomes of patients with atrial fibrillation: a report of the American College of Cardiology/American Heart Association task force on clinical data standards (writing committee to develop data standards on atrial fibrillation). Circulation 2004:109:3223-43.

3 Kato T, Yamashita T, Sagara K, et al. Progressive nature of paroxysmal atrial fibrillation. Observations from a 14 year follow-up study. Circ $J$ 2004;68:568-72

4 Gaynor SI, Prasad S, Cox JL, et al. The long-term outcome of patients with coronary disease and atrial fibrillation undergoing the Cox-Maze procedure. Presented at the $83^{\text {rd }}$ Annual Meeting of the American Association for Thoracic Surgery, Boston, Massachusetts, May 2003 (referenced in reference 6 below)

5 Cox JL, Schuessler RB, Lappas DG, et al. An $81 \frac{1}{2}$ year clinical experience with surgery for atrial fibrillation. Ann Surg 1996;224:267-75.

6 Cox JL. Cardiac surgery for arrhythmias. J Cardiovasc Electrohpysiol 2004; 15:250-62

7 McCarthy PM, Gillinov AM, Castle L, et al. The Cox-Maze procedure: the Cleveland Clinic experience. Semin Thorac Cardiovasc Surg 2000;12:25-9.

8 Arcidi JM, Doty DB, Millar RC. The Maze procedure: the LDS Hospital experience. Semin Thorac Cardiovasc Surg 2000;12:38-43.

9 Gaita F, Riccardi R, Caponi D, et al. Linear cryoablation of the left atrium versus pulmonary vein cryoisolation in patients with permanent atrial fibrillation and valvular heart disease: correlation of electroanatomic mapping and long term clinical results. Circulation 2005;111:136-42.

10 Hsu L-F, Jaïs P, Sanders $P$, et al. Catheter ablation of permanent atrial fibrillation in the elderly. Heart Rhythm 2005;2:1S-P6-64

11 Pappone C, Santinelli V. The who, what, why, and how-to guide for circumferential pulmonary vein ablation. J Cardiovasc Electrophysiol 2004; 15:1226-30.

12 Mandeep B, Marrouche NF, Martin DO, et al. Impact of age on the outcome of pulmonary vein isolation for atrial fibrillation using circular mapping technique and cooled-tip ablation catheter: a retrospective analysis. J Cardiovasc Electrophysiol 2004;15:8-13.

13 Ouyang F, Ernst $S$, Antz $M$, et al. The important role of complete pulmonary vein isolation in catheter ablation of permanent atrial fibrillation. Heart Rhythm 2005;2:1S-P3-64.

14 Jalife J. Rotors and spiral waves in atrial fibrillation. J Cardiovasc Electrophysiol 2003;14:776-80.

15 Allessie M, Ausuma J, Schotten U. Electrical, contractile and structural remodelling during atrial fibrillation. Cardiovasc Res 2002;54:230-46.

16 Fuller IA, Wood MA. Intramural coronary vasculature prevents transmural radiofrequency lesion formation. Circulation 2003;107:1797-803.

17 Knaut M, Tugtekin SM, Matschke K. Pulmonary vein isolation by microwave energy ablation in patients with permanent atrial fibrillation. J Card Surg 2004; 19:211-5.

18 Wisser W, Khazen C, Deviatko E, et al. Microwave and radiofrequency ablation yield similar success rates for treatment of chronic atrial fibrillation. Eur J Cardiothoracic Surg 2004;25:101 1-7.

19 Doukas G, Samani NJ, Alexiou C, et al. Left atrial radiofrequency ablation during mitral valve surgery for continuous atrial fibrillation: results of a prospective randomised clinical trial. Heart 2005:91(suppl I):A1

20 Verma A, Kilicaslan F, Pisano E, et al. Response of atrial fibrillation to pulmonary vein isolation is directly related to resumption and delay of pulmonary vein conduction. Circulation 2005;1 12:627-35

21 Sanders $\mathbf{P}$, Jaiis $P$, Hocini $M$, et al. Electrical disconnection of the coronary sinus by radiofrequency catheter ablation to isolate a trigger of atrial fibrillation. J Cardiovasc Electrophysiol 2004;15:364-8.

22 Ouyang F, Bansch D, Ernst $S$, et al. Complete isolation of left atrium surrounding the pulmonary veins: new insights from the double-Lasso technique in paroxysmal atrial fibrillation. J Am Coll Cardiol 2003;42: 1271-82.

23 Hoyt RH, Wood M, Daoud E, et al for the US CryoCor Investigators. Transvenous catheter cryoablation for treatment of atrial fibrillation: results of a feasibility study. J Am Coll Cardiol 2003;42:752-8.

24 Tse HF, Reek S, Timmermans C, et al. Pulmonary vein isolation using transvenous catheter cryoablation for treatment of atrial fibrillation without risk of pulmonary vein stenosis. J Interv Card Electrophysiol 2004;11:117-26.

25 Hocini $M$, Sanders $P$, Jaïs $P$, et al. Techniques for curative treatment of atrial fibrillation. J Cardiovasc Electrophysiol 2004;15:1467-71.

26 Becker AE. Left atrial isthmus: anatomic aspects relevant for linear catheter ablation procedures in humans. J Cardiovasc Electrophysio 2004; 15:809-812.

27 HRS website. http://www.hrsonline.org/swPositionStaementFiles/ ps99316504.asp.

28 Pappone C, Rosanio S, Augello G, et al. Mortality, morbidity and quality of life after circumferential pulmonary vein ablation for atrial fibrillation. Outcomes from a controlled nonrandomised long-term study. J Am Coll Cardiol 2003:42: 185-97.

\section{IMAGES IN CARDIOLOGY}

Left ventricular pseudoaneurysm complicating acute myocardial infarction: improved diagnosis by real time three dimensional echocardiography

A

59 year old man was admitted to our hospital with an inferoposterolateral acute myocardial infarction. Primary stenting was performed on an occluded first obtuse marginal coronary artery. Left ventriculography showed contrast leakage through the inferior wall suggesting the existence of a left ventricular pseudoaneurysm (left panel).

Transthoracic two dimensional echocardiography was also performed, but it did not show a hint of the pseudoaneurysm, nor of its neck.

However, transthoracic real time three dimensional echocardiography (Sonos 7500, Philips Medical Systems) demonstrated a clear view of the pseudoaneurysm neck, with a short intraventricular path through the inferior wall of the left ventricle (right panel).
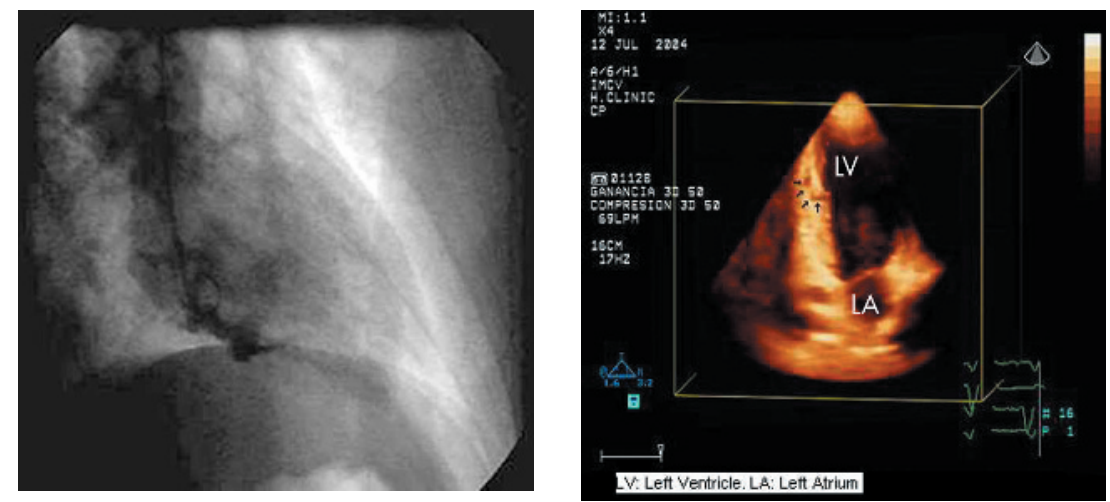

X Freixa

M Sitges

C Paré

xavifreixa@menta.net 